\title{
Entropy of geoelectrical time series in the natural time domain
}

\author{
A. Ramírez-Rojas ${ }^{1}$, L. Telesca ${ }^{2}$, and F. Angulo-Brown ${ }^{3}$ \\ ${ }^{1}$ Departamento de Ciencias Básicas, Universidad Autónoma Metropolitana, Av. San Pablo 180, Col. Reynosa, Azcapotzalco, \\ México D. F. 02200, México \\ ${ }^{2}$ National Research Council, Institute of Methodologies for Environmental Analysis, C. da S. Loja, 85050 Tito (PZ), Italy \\ ${ }^{3}$ Departamento de Física, Escuela Superior de Física y Matemáticas, Instituto Politécnico Nacional, Edif. 9, U.P. Zacatenco, \\ 07738, México D.F., México
}

Received: 8 September 2010 - Accepted: 3 December 2010 - Published: 28 January 2011

\begin{abstract}
Seismic electric signals (SES) have been considered precursors of strong earthquakes, and, recently, their dynamics have been investigated within the Natural Time Domain (NTD) (Varotsos et al., 2004). In this paper we apply the NTD approach and the chaotic map signal analysis to two geoelectric time series recorded in a seismically very active area of Mexico, where two strong earthquakes, $M=6.6$ and $M=7.4$, occurred on 24 October 1993 and 14 September 1995, respectively. The low frequency geoelectric signals measured display periods with dichotomic behavior. Our findings point out to an increase of the correlation degree of the geoelectric signals before the occurrence of strong earthquakes; furthermore, the power spectrum and entropy in NTD are in good agreement with the results published in literature. Our results were validated by the analysis of a chaotic map simulated time series, which revealed the typical characteristics of artificial noise.
\end{abstract}

\section{Introduction}

The complexity of the Earth's crust and the presence of criticality in seismicity (Uyeda et al., 2009), does not allow performing earthquake (EQ) prediction solely using seismological tools. Thus, many researchers have focused their attention on other type of tools, i.e., signals shortly preceding the earthquake's occurrence. Among the several geophysical quantities, which could reveal links to tectonic activity, the geoelectric field has assumed a relevant role (Hayakawa, 2006). Some mechanisms associated with the generation of electric signals (Vallianatos and Triantis, 2008) when materials are under stress conditions are mainly the piezoelectric effect (Nitsan, 1977), the electrokinetic effects (Ishido and

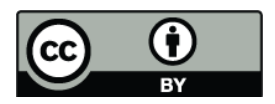

Correspondence to: A. Ramírez-Rojas (arr@ correo.azc.uam.mx)
Mizutani, 1981), the point defects (Varotsos and Alexopoulos, 1986), the emission of electrons (Brady and Rowell, 1986), and the motion of charged dislocations (Molchanov and Hayakawa, 1995). It was observed that geoelectric signals measured in seismic areas behave as white noise during low seismic activity, but before the occurrence of strong EQs they behave as 1/f-noise (Ramirez-Rojas et al., 2004). A link between the dynamical patterns of geoelectric fluctuations and the EQ mechanism of preparation was proposed by (Ramírez-Rojas et al. 2008). Seismic Electric Signals $(\mathrm{SES})$ are low frequency $(\leq 1 \mathrm{~Hz})$ transient anomalies of geoelectric signals (Varotsos et al., 2001, 2002, 2003) generated when the stress reaches a critical value in the EQ focal area (Varotsos et al., 2001). SES activity has been observed in geoelectric time series recorded in seismically active regions and appeared before the occurrence of large EQs with lead times ranging from several hours to a few months before the main shock (Varotsos et al., 2002). The first SES were reported in Greece (Varotsos, and Alexopoulos 1984a; Varotsos and Alexopoulos, 1984b), in Japan (Uyeda et al., 2000) and México (Flores-Márquez et al., 2007). The features that distinguish SES activity are dichotomic nature and long-range correlations (Varotsos et al., 2001, 2002, 2003). Dichotomous behavior can be detected in a variety of physical systems (Lorito et al., 2005; Abich et al., 2004; Mercik and Weron, 2001; Ramirez-Rojas et al., 2004).

The Natural Time Domain (NTD) approach, firstly developed by Varotsos et al. (2001, 2003), enables us to follow the dynamical evolution of a system and identify when it enters into a critical stage (Abe et al., 2005). This approach may constitute a novel contribution to EQ short-term prediction (Uyeda et al., 2009). The effectiveness of the NTD method in distinguishing SES from artificial noises (AN) was shown in Varotsos et al. (2004), where AN are dichotomous electrical disturbances recorded at a measuring site due to the nearby man-made electric sources. 

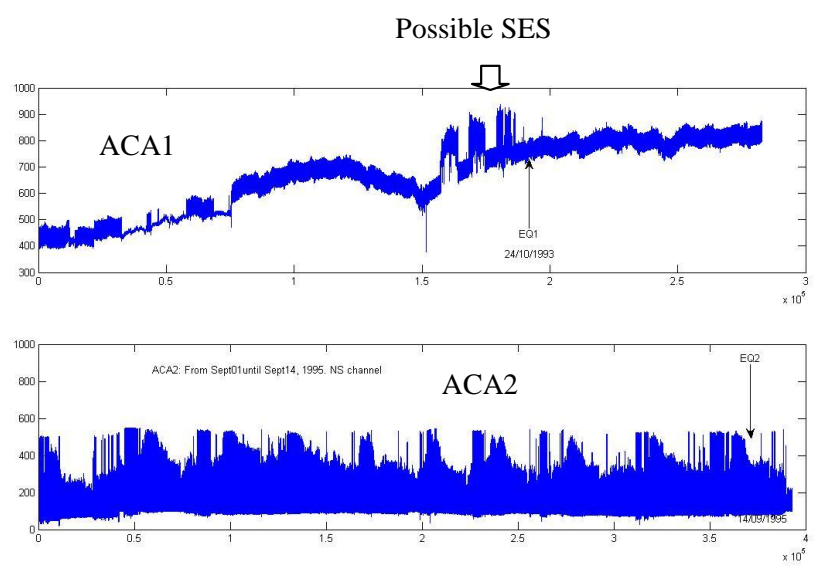

Fig. 1. Acapulco time series. The thin downward arrows indicate the EQ1 and EQ2 occurrence.

In the present paper we analyze the dichotomic behavior observed in geoelectric time series recorded in the GuerreroOaxaca region in the Mexican Pacific coast appearing a few weeks before two strong earthquakes, EQ1 $[M=6.6$, 24 October 1993, $\left.\left(16.54^{\circ} \mathrm{N}, 98.98^{\circ} \mathrm{W}\right)\right]$ and EQ2 $[M=$ 7.4, 14 September $\left.1995,\left(16.31^{\circ} \mathrm{N}, 98.88^{\circ} \mathrm{W}\right)\right]$. These data, previously analyzed using several other nonlinear methods (Ramírez-Rojas et al., 2008; Ramirez-Rojas et al., 2004; Telesca et al., 2008, 2009a, b; Hernández-Pérez et al., 2010), will be investigated by means of the NTD approach. Furthermore, the obtained results will be compared with ionic dichotomic simulations based on the Liebovitch and Thot chaotic model (Lievbovitch and Thot, 1991). The temporal correlations of the experimental and simulated time series were investigated by the DFA method (Peng et al., 1994). The present paper is organized as follows: In Sect. 2 the data set acquisition is described; Sect. 3 is devoted to the methods of analysis; in Sect. 4 the results and Sect. 5 the concluding remarks are presented.

\section{Data}

The monitored area is located along the South Pacific Mexican coast, near the Middle American trench, which is the border between the Cocos and American tectonic plates where large earthquakes have been generated. This region is characterized by high seismic activity and is constituted of composite terrains with both undersea volcanic and sedimentary sequences (Angulo-Brown et al., 1998). The monitoring station was located at $\left(16^{\circ} 50^{\prime} \mathrm{N}, 99^{\circ} 47^{\prime} \mathrm{W}\right)$ close to Acapulco city (Yépez et al., 1995). The experimental set-up was based on the VAN methodology (Varotsos et al., 1984a, b), in which two geoelectric fluctuations $(\Delta \mathrm{V})$ were recorded between two electrodes buried $2 \mathrm{~m}$ into the ground and $50 \mathrm{~m}$ apart, with sampling times $\Delta \mathrm{t}=2 \mathrm{~s}$ and $\Delta \mathrm{t}=4 \mathrm{~s}$, respectively. In order to remove all the high frequency noise, the sam-
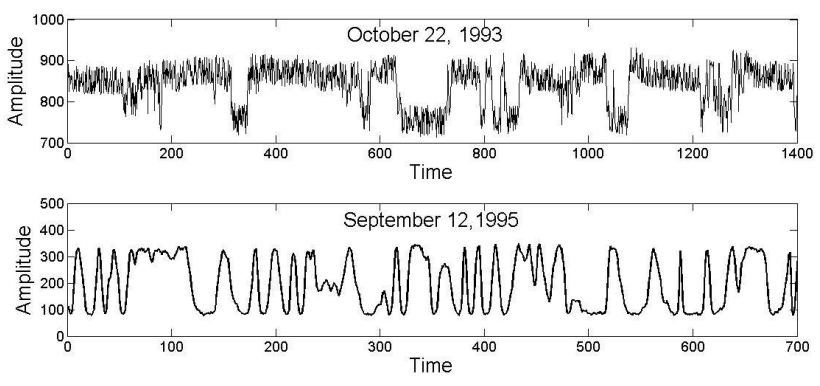

Fig. 2. Excerpts of the analyzed time series displaying dichotomous features: (a) SES activity in 22 October 1993 in ACA1. (b) SES activity in 12 September 1995 (ACA2).

pled data were filtered by a low-pass filter in the range: $0<f<0.125 \mathrm{~Hz}$.

The signals are shown in Fig. 1. Both signals (ACA1 and ACA2) are characterized by SES activity before the occurrence of EQ1 and EQ2 respectively. Excerpts of both time series show dichotomic behavior of both signals (Fig. 2).

\section{Methods}

\subsection{Detrended Fluctuation Analysis (DFA)}

The DFA (Peng et al., 1994) permits the detection of longrange correlations embedded in a seemingly non-stationary time series, and avoids the spurious detection of apparent long-range correlations that are artifacts of non-stationarity. The DFA has been widely applied in many scientific fields, and in particular to investigate earthquakes and earthquakerelated phenomena (Telesca et al., 2003, 2004a, b; Telesca and Lovallo, 2009). The DFA method is briefly described as follows. Given the time series $\{x(k)\}$ a new time series $\{y(k)\}$ is obtained by integration, $y(k)=\sum_{i=1}^{k}\left(x(i)-x_{\text {ave }}\right)$ where $x_{\text {ave }}$ indicates the average of $\{x(k)\}$. Next, the integrated time series is divided into boxes of equal length $n$. For each box of length $n$, a least-squares line is fitted to the data, (representing the linear trend in that box, $y_{n}(k)$ ). In each box the integrated time series is detrended, $y(k)-y_{n}(k)$, and the root mean-square fluctuation of this integrated and detrended time series is calculated:

$F(n)=\sqrt{\frac{1}{N} \sum_{k=1}^{N}\left[y(k)-y_{n}(k)\right]^{2}}$.

In order to provide a relationship between $F(n)$ and the box size $n$, the calculation is repeated over all the available time scales (box sizes). Typically $F(n)$ will increase with box size $n$. A linear relationship on log-log graph indicates the presence of scaling:

$F(n) \propto n^{\alpha}$. 

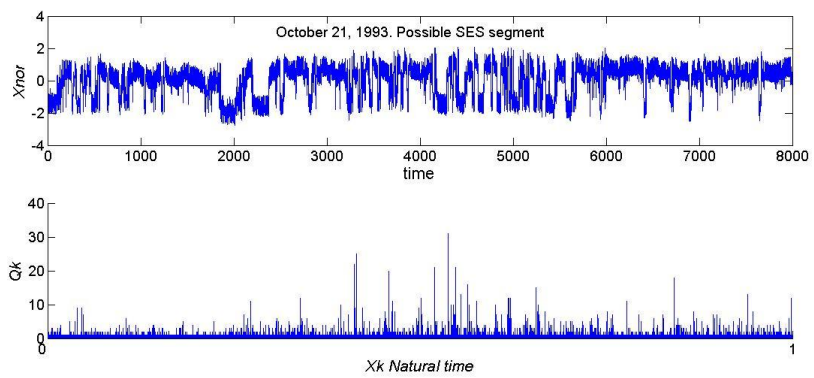

Fig. 3. Excerpt of ACA1 on 21 October 1993, in conventional time (top) and in NTD (bottom).

The value of the scaling exponent $\alpha$ characterizes the correlation of the time series. A white noise has $\alpha=0.5 ; \alpha=1$ corresponds to $1 / f$ noise and $\alpha=1.5$ to the Brownian noise. If $0.5<\alpha \leq 1$ persistent long-range power-law correlations exist. In contrast, $0<\alpha<0.5$ indicates antipersistent powerlaw correlation (Telesca et al., 2009).

\subsection{Natural Time Domain (NTD)}

The NTD method (Varotsos et al., 2001, 2002) works as follows: Given a time series of $N$ pulses, the natural time is defined as $\chi_{k}=k / N$. $Q_{k}$ stands for the duration of the $k$-th pulse. For a dichotomous time series, $Q_{k}$ represent the dwell time of the $k$-th pulse. A representation in natural time of a segment of dichotomous noise is shown in Fig. 3.

In NTD the aim is to study the evolution of the pair $\left(\chi_{k}, Q_{k}\right)$. The normalized power spectrum $\Pi(\omega) \equiv$ $|\Phi(\omega)|^{2}$ in NTD is defined as (Varotsos et al., 2001, 2002):

$\Phi(\omega)=\sum_{k=1}^{N} p_{k} \exp \left(i \omega \frac{k}{N}\right)=\sum_{k=1}^{N} p_{k} \exp \left(i \omega \chi_{k}\right)$

where

$p_{k}=\frac{Q_{k}}{\sum_{k=1}^{N} Q_{k}}$.

In Eq. (3) $\omega=2 \pi \phi$, where $\phi$ is the natural frequency. For $0<\phi<0.5$, $\Pi(\omega)$ (or $\Pi(\phi)$ ) becomes the characteristic function for the probability distribution of $p_{k}$ (Stratonovich, 1963). For SES activities, since the system is in a critical stage, Varotsos et al. (2001, 2002) deduced the following expression:

$\Pi_{S E S}(\omega)=\frac{18}{5 \omega^{2}}-\frac{6 \cos \omega}{5 \omega^{2}}-\frac{12 \sin \omega}{5 \omega^{3}}$.

In the limit $\omega \rightarrow 0$, Eq. (5) tends to $\Pi_{\mathrm{SES}}(\omega) \approx 1-0.07 \omega^{2}$, where the variance of $\chi$ is given by $\kappa_{1}$ SES $=<\chi^{2}>-<$ $\chi>^{2}=0.07$. Varotsos et al. (2003) obtained for ion current fluctuations in membrane channels $\kappa_{1}=0.080$, for artificial noises $\kappa_{1}>0.083$ and for a uniform distribution of $p_{k}$ $\kappa_{1} \approx 0.083$.
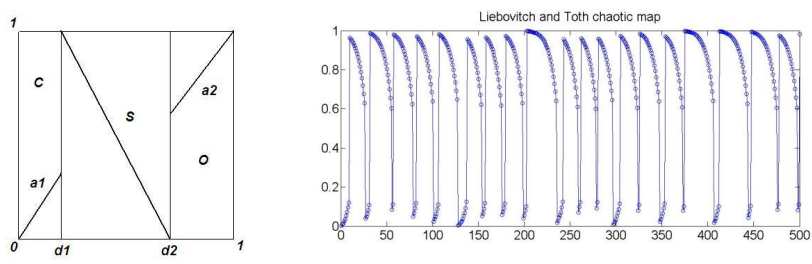

Fig. 4. Liebovitch and Thot map and an excerpt of the generated time series.

The entropy is a concept equally applicable to deterministic as well as stochastic processes. In NTD the entropy is defined as (Varotsos et al., 2001, 2003):

$\mathrm{S} \equiv<\chi \ln \chi>-<\chi>\ln <\chi>$

where $\langle\chi\rangle=\sum_{i=1}^{N} p_{i} \chi_{i}$ and $\langle\chi \ln \chi\rangle=\sum_{i=1}^{N} p_{i} \chi_{i} \ln \chi_{i}$. By means of the entropy in NTD, SES activities in geoelectric signals were discriminated from artificial noises (Varotsos et al., 2001, 2003); it was found that $\mathrm{S}_{\mathrm{SES}}<\mathrm{S}_{\mathrm{u}}<\mathrm{S}_{\mathrm{AN}}$, where $\mathrm{S}_{\mathrm{u}} \approx 0.0966$ is the entropy of a uniform distribution (Sarlis et al., 2008).

\subsection{Liebovitch and Thot chaotic map}

This Liebovitch and Toth (LT) map was introduced as a deterministic chaotic model to study the ionic channels dynamics (Lievobitch and Thot, 1991):

$f\left(x_{n+1}\right)= \begin{cases}a_{1} x_{n} & 0 \ll x_{n}<d_{1} \\ \frac{d_{2}-x_{n}}{d_{2}-d_{1}} & d_{1} \leq x_{n} \leq d_{2} \\ a_{2}\left(x_{n}-1\right)+1 & d_{2}<x_{n} \leq 1\end{cases}$

In Fig. 4 the map and an excerpt of the time series generated by the map are shown. Some of their dynamical properties have been studied in (Lievbovitch and Thot, 1991; MuñozDiosdado et al., 2005).

\section{Results}

We analyzed two geoelectric time series: ACA1, which corresponds to a possible SES activity in EW channel observed five days before EQ1 (epicenter located approximately $80 \mathrm{~km}$ far from the station), and ACA2, which is a dichotomous segment recorded in NS channel some days before EQ2 (epicenter located about $112 \mathrm{~km}$ far from the station) (Fig. 1). We also analyzed the time series generated by means of the LT chaotic map (Muñoz-Diosdado et al., 2005).

\subsection{Correlation analysis (DFA)}

The values of the $\alpha_{\mathrm{DFA}}$ exponent (Eq. 2) for ACA1 and ACA2 are shown in Figs. 5 and 6 respectively. The time 


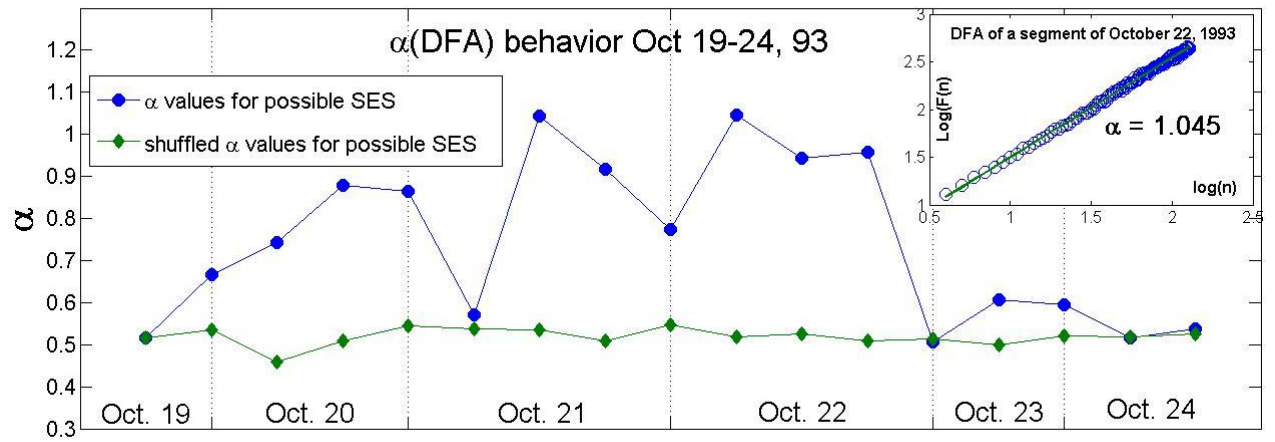

Fig. 5. Time variation of $\alpha_{\text {DFA }}$ for ACA1 SES activity (blue circles). Time variation of $\alpha_{\text {DFA }}$ for shuffled ACA1 time series (green diamonds). Inset: $\mathrm{F}(\mathrm{n}) \sim \mathrm{n}$ relationship for ACA1 measured on 22 October 1993: the scaling behaviour indicates the presence of long-range correlations. EQ1 occurred on 24 October 1993.
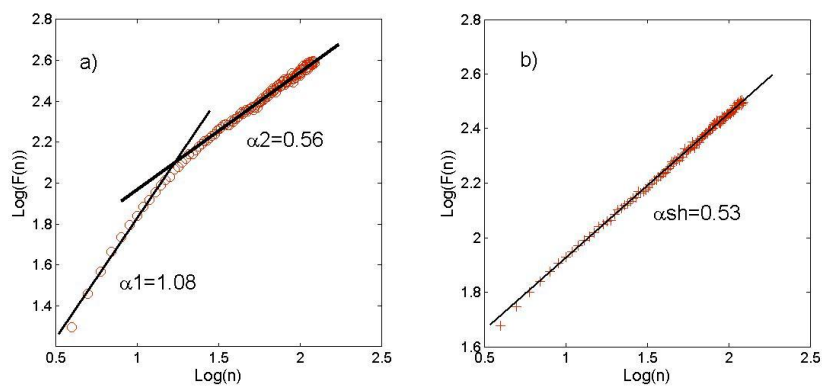

Fig. 6. An example of $\mathrm{F}(\mathrm{n}) \sim \mathrm{n}$ relationship for ACA2 measured on 1 September 1995. (a) Two scaling regions can be detected, with $1 / f$ dynamics at short scales and white noise dynamics at long scales. (b) $\mathrm{F}(\mathrm{n}) \sim \mathrm{n}$ relationship for the shuffled series ACA2 measured on 1 September 1995: the correlations are completely destroyed by the shuffling procedure and the scaling exponent is about 0.5 .
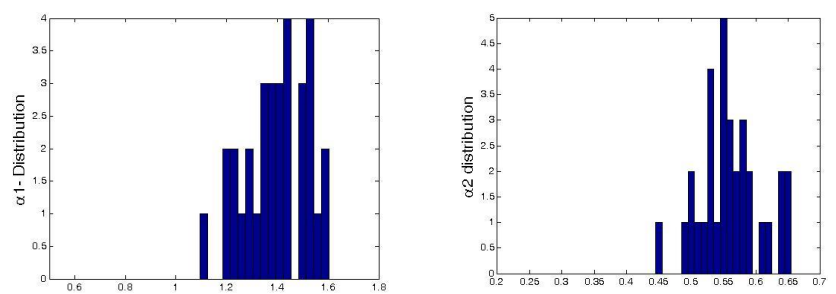

Fig. 7. Distribution of $\alpha_{1}$-values (left panel) and $\alpha_{2}$-values (right panel).

series were divided into non-overlapping windows of $6 \mathrm{~h} \mathrm{du}-$ ration. In each window the scaling exponents were calculated as the slope of the line fitting the $\mathrm{F}(\mathrm{n}) \sim \mathrm{n}$ relationship, plotted in $\log -\log$ scales, by a least-square method. Also the value of the scaling exponents calculated for randomly shuffled series was calculated and shown in Figs. 5 and 6. For ACA1 $\alpha_{\text {DFA }}$ increases from $\approx 0.5$ (on 19 October) up $\approx 1.045$ (on 22 October). After 23 and 24 October, $\alpha_{\text {DFA }}$ decreases $\approx 0.58$
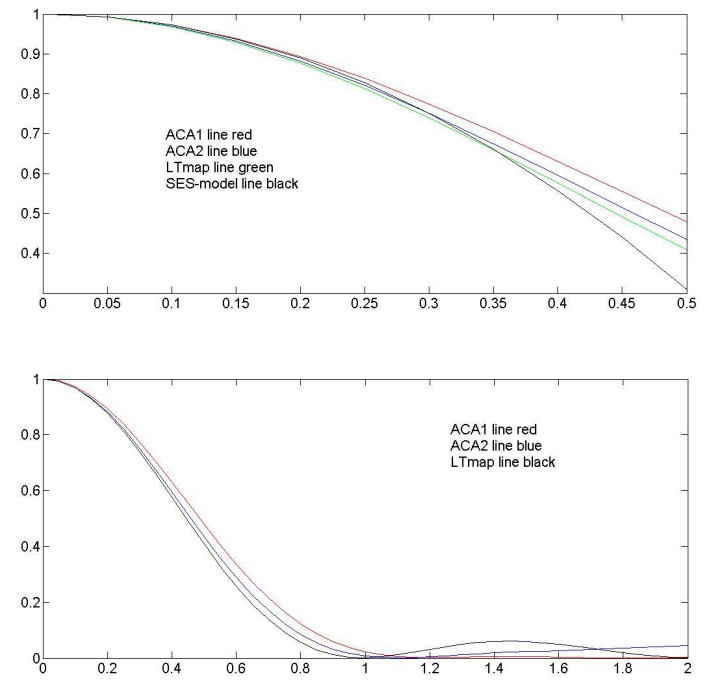

Fig. 8. Power spectrum calculated in the NTD.

(blue circles in Fig. 5). The exponent $\alpha_{\mathrm{DFA}} \approx 0.5$ for the randomly shuffled series (green diamonds in Fig. 5), and this indicates that the scaling observed depends on the long-range correlation and not on the broad probability density function (Kantelhardt et al., 2002) measured.

For ACA2 a crossover appears, indicating the co-existence of two different dynamics, a flicker-noise $(\sim 1 / f)$ behavior at small scales and a white noise behavior at long scales. Figure 6a shows as an example the $\mathrm{F}(\mathrm{n}) \sim \mathrm{n}$ relationship for ACA2 measured on 1 September 1995: two different scaling exponents can be estimated, $\alpha_{1} \approx 1$ at short scales and $\alpha_{1} \approx 0.5$ at long scales. Such a crossover disappears when the series has been shuffled, with a scaling exponent of about 0.5 (Fig. 6b).

The distributions of $\alpha_{1}$ and $\alpha_{2}$ are shown in Fig. 7. Such crossovers in the $\mathrm{F}(\mathrm{n}) \sim \mathrm{n}$ relationship were also obtained by Varotsos et al. (2002) for SES recorded in Greece. 


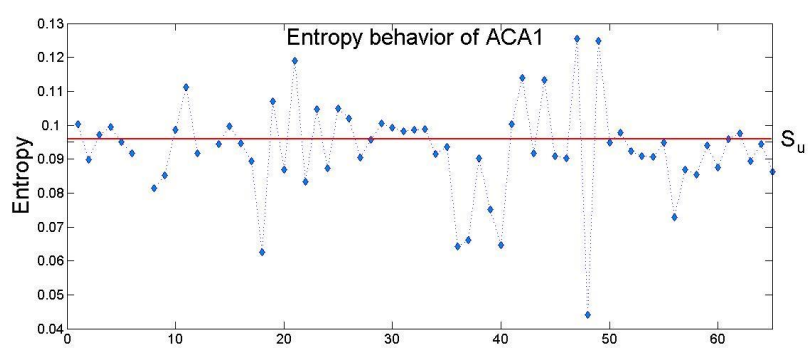

a)

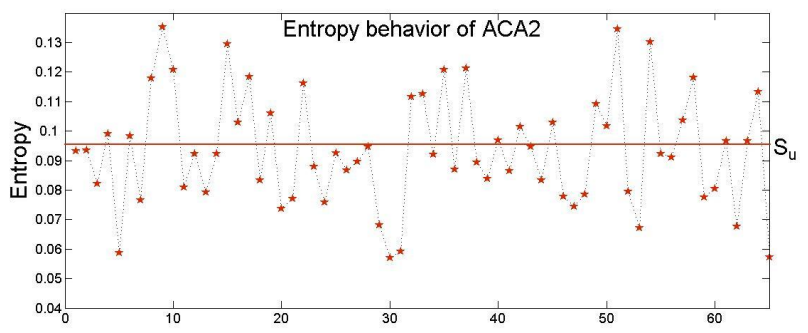

b)

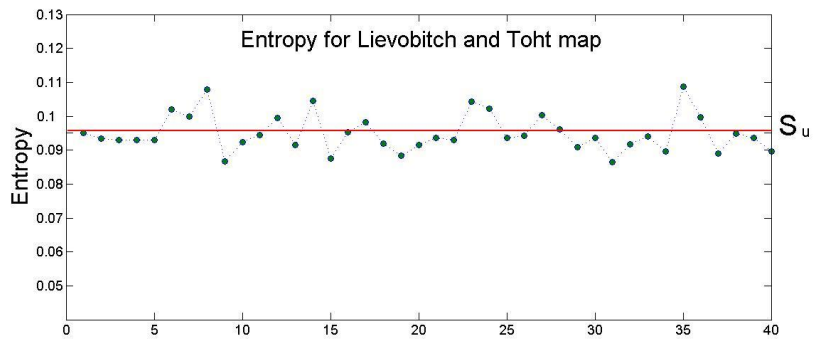

c)

Fig. 9. Entropy in NTD: (a) ACA1, (b) ACA2, (c) LT-map in the chaotic region.

\subsection{Natural time domain analysis}

By means of Eq. (3) the power spectrum in NTD of ACA1 $\left(\Pi(\omega)_{\mathrm{ACA} 1}\right), \mathrm{ACA} 2\left(\Pi(\omega)_{\mathrm{ACA}}\right)$ and the LT-map generated signal $\left(\Pi(\omega)_{\mathrm{LT}}\right)$ was calculated and then compared with theoretical model of SES in the limit $\omega \rightarrow 0, \Pi_{\mathrm{SES}}(\omega) \approx 1-$ $0.07 \omega^{2}$ (Fig. 8). Both $\Pi(\omega)_{\text {ACA1 }}$ and $\Pi(\omega)_{\text {ACA2 }}$ are above $\Pi(\omega)_{\mathrm{LT}}$. These results are in agreement with Varotsos et al. (2003).

In Fig. 9 the NTD entropy calculated by means of Eq. (6) is shown for the three signals. For ACA1 (Fig. 9a) we obtain $\mathrm{S}_{\mathrm{ACA} 1}=0.0898 \pm 0.0139$; for ACA2 (Fig. 9b) we obtain $\mathrm{S}_{\mathrm{ACA} 2}=0.0934 \pm 0.0191$ and for the LT-map signal (Fig. 9c) $\mathrm{S}_{\mathrm{LT}}=0.09494 \pm 0.00548$. Since the uniform distribution entropy reported by Varotsos et al. (2003) is characterized by $\mathrm{S}_{\mathrm{u}}=0.0967$, it follows that $\mathrm{S}_{\mathrm{ACA} 1} \leq \mathrm{S}_{\mathrm{ACA} 2} \leq \mathrm{S}_{\mathrm{LT}} \leq \mathrm{S}_{\mathrm{u}}$. Although possible SES signals could be very noisy, they have NTD entropies below $S_{u}$ and also below most of the ANentropies reported by Varotsos et al. (2003). That is, in terms of the entropic behavior they are in the range of SESentropies. In particular for ACA1 the S-values lower than 0.07 correspond with the occurrence of anomalies showed in Fig. 1a. For the LT-map in the chaotic region, the S-values behave as an artificial-like noise.

Furthermore, we calculated the variance $\kappa_{1}$ of the signals and obtained for ACA1 $<\kappa_{1}>=0.0757 \pm 0.0052$ and for ACA2 $<\kappa_{1}>=0.0798 \pm 0.011$, and both of them are smaller than the variance of the uniform distribution $\left(\kappa_{1}=0.0833\right)$, in agreement with Varotsos et al. (2003). We clarify that as far as the entropy is concerned, the present investigation referred only to the S-values which correspond to the entropy in natural time when the time-series is analyzed in forward time (e.g., Varotsos et al., 2003, 2005a). Natural time, however, allows also the definition of an entropy S-upon reversing the time arrow (Varotsos et al., 2005b, 2006), which interestingly obeys a similar property, i.e., $\mathrm{S} \leq \mathrm{Su}$ for critical systems as the SES activities. This is a challenging point that will be the objective of future research.

\section{Concluding remarks}

In this work we have analyzed three signals of dichotomic nature, two of them (ACA1 and ACA2) are geoelectric signals measured at the Acapulco station in Mexico and associated with the occurrence of two strong EQs occurring on 24 October $1993(M=6.6)$ and 14 September $1995(M=7.4)$, respectively. The third signal is a time series generated by the Liebovitch and Thot chaotic map. The DFA results reveal a significant (respect to random shuffles of the series) enhancement of their temporal correlation before the occurrence of the two EQs. In particular, the time variation of the ACA1 DFA scaling exponent reveals a succession of different dynamical states from white noise to a long-range correlation structure and again white noise, suggesting that the system enters into a critical stage when the ACA1 is characterized by the long-range correlation structure. The ACA2 DFA scaling behavior is characterized by two dynamical behaviors with a crossover, consistent with the results reported in FloresMárquez et al. (2007) and Ramirez-Rojas et al. (2004). In the NTD, the spectrum of the geoelectric signals are below of that of the SES theoretical model and LT-map. We consider our results to contribute to the development of the NTD method as a novel analysis tool for signals of dichotomic nature.

Acknowledgements. A. R. R. thanks for the financial support received by Departamento de Ciencias Básicas de la UAMAzcapotzalco. L. T. acknowledges the financial support received by CNR and CONACYT within the frame of the CNR-CONACYT Bilateral Agreement for Scientific and Technological Cooperation. F. A. B. thanks for partial support from COFAA and EDI-IPN.

Edited by: C.-V. Meister

Reviewed by: two other anonymous referees 


\section{References}

Abe, S., Sarlis, N. V., Skordas, E. S., Tanaka H. K., and Varotsos, P. A.: Origin of the Usefulness of the Natural-Time Representation of Complex Time Series Phys. Rev. Lett., 94, 170601, doi:10.1103/PhysRevLett.94.170601, 2005.

Abich, K., Keil, A., Reiss, D., Wunderlich, Ch., Neuhauser, W., and Toschek, P. E.: Thermally activated hopping of two ions trapped in a bistable potential well, J. Opt. B.-Quantum. S. O., 6, 3, 2004.

Angulo-Brown, F., Ramírez-Guzmán, A. H., Yépez, E., RudolfNavarro A., and Pavía-Miller, C. G.: Fractal geometry and seismicity in the Mexican subduction zone, Geofísica Internacional, 37, 29-33, 1998.

Brady, B. T. and Rowell, G. A.: Laboratory investigation of the electrodynamics of rock fracture, Nature, 321, 488-492, 1986.

Flores-Márquez, L., Márquez-Cruz, J., Ramírez-Rojas, A., GálvezCoyt, G., and Angulo-Brown, F.: A statistical analysis of electric self-potential time series associated to two 1993 earthquakes in Mexico, Nat. Hazards Earth Syst. Sci., 7, 549-556, doi:10.5194/nhess-7-549-2007, 2007.

Hayakawa M.: Natural Electromagnetic Phenomena and Electromagnetic Theory, Investigating R\&D Committee on Natural Electromagnetic Phenomena and Electromagnetic Theory, Japan, 1-25, 2006.

Hernández-Pérez, R., Guzmán-Vargas, L., Ramírez-Rojas, A., and Angulo-Brown, F.: Pattern synchrony in electrical signals related to earthquake activity, Physica A, 389, 1239-1252, 2010.

Ishido, T. and Mizutani, H.: Experimental and Theoretical Basis of Electrokinetic Phenomena in Rock-Water Systems and Its Applications to Geophysics, J. Geophys. Res., 86, 1763-1775, 1981.

Kantelhardt, J. W., Zschiegner, S. A., Konscienly-Bunde, E., Havlin, S., Bunde, A., and Stanley, H. E.: Multifractal detrended fluctuation analysis of nonstationary time series, Physica A, 2002, 316, 87-114, 2002.

Lievbovitch, S. L. and Thot, T. I.: A model of ion channel kinetics using deterministic chaotic rather than stochastic processes, J. Theor. Biol., 148, 243-267, 1991.

Lorito, S., Schmitt, D., Consolina, G., and De Michelis, P.: Stochastic resonance in a bistable geodynamo model, Astron. Nachr./AN 326, No. 3/4, 227-230, doi:10.1002/asna.200410381, 2005.

Mercik, S. and Weron, K.: Stochastic origins of the long-range correlations of ionic current fluctuations in membrane channels, Phys. Rev E, 63, 051910, 2001.

Molchanov, O. A. and Hayakawa, M.: Generation of ULF electromagnetic emissions by microfracturing, Geophys. Res. Lett., 22, 3091-3094, 1995.

Muñoz-Diosdado, A., Guzman-Vargas, L., Ramírez-Rojas, A., del Río-Correa, J. L., and Angulo-Brown, F.: Some cases of crossover behavior in Heart interbeat and electroseismic time series, Fractals, 13, 1-11, 2005.

Nitsan U.: Electromagnetic emission accompanying fracture of quartz-bearing rocks, Geophys. Res. Lett., 4, 333-336, 1977.

Peng C.-K., Buldyrev S. V., Havlin S., Simons M., Stanley H. E., and Goldberger A. L.: Mosaic organization of DNA nucleotides, Phys. Rev. E, 49, 1685, 1994.

Ramirez-Rojas, A., Pavia-Miller, C. G., and Angulo-Brown, F.: Statistical behavior of the spectral exponent and the correlation time of electric self-potential time series associated to the Ms $=7.4$ Sept. 14th, 1995 earthquake in Mexico, Phys. Chem. Earth, 29, 305, 2004.
Ramírez-Rojas, A., Flores-Márquez, E. L., Guzmán-Vargas, L., Gálvez-Coyt, G., Telesca, L., and Angulo-Brown, F.: Statistical features of seismoelectric signals prior to M7.4 GuerreroOaxaca earthquake (Mxico), Nat. Hazards Earth Syst. Sci., 8, 1001-1007, doi:10.5194/nhess-8-1001-2008, 2008.

Sarlis, N. V., Skordas, E. S., Lazaridou, M. S., and Varotsos, P. A.: Investigation of seismicity after the initiation of a Seismic Electric Signal activity until the main shock, Proc. Jpn. Acad. Ser. B, 84, 331-343, 2008.

Stratonovich, R. L.: Topics on the Theory of Random Noise (Gordon and Breach, New York, 1, 3-10, 1963.

Telesca, L., Lapenna, V., and Macchiato, M.: Investigating the time-clustering properties in seismicity of Umbria-Marche region (central Italy), Chaos Solitons \& Fractals, 18, 203-217, 2003.

Telesca, L., Lapenna, V., Lovallo, M., and Macchiato, M.: Longrange time-correlation properties of seismic sequences, Chaos Solitons \& Fractals, 21, 387-393, 2004a.

Telesca, L., Cuomo, V., Lapenna, V., and Macchiato, M.: Detrended fluctuation analysis of the spatial variability of the temporal distribution of Southern California seismicity, Chaos Solitons \& Fractals 21, 335-342, 2004b.

Telesca, L. and Lovallo, M.: Non-uniform scaling features in central Italy seismicity: A non-linear approach in investigating seismic patterns and detection of possible earthquake precursors, Geophys. Res. Lett., 36, L01308, doi:10.1029/2008GL036247, 2009.

Telesca, L., Lovallo, M., Ramirez-Rojas, A., and Angulo-Brown, F.: A nonlinear strategy to reveal seismic precursory signatures in earthquakes-related self-potential signals, Physica A, 388, 11811186, 2009a.

Telesca, L., Lovallo, M., Ramirez-Rojas, A., and Angulo-Brown, F.: Analyzing scaling behavioral trends in self-potential earthquakerelated data measured in Mexico, Int. J. Nonlinear Sci., 8, 3, 279 283, 2009b.

Telesca, L., Lovallo, M., Ramirez-Rojas, A., and Angulo-Brown, F.: Non-uniform scaling behavior in self-potential earthquakerelated signals, Fluct. Noise Lett., 8, 3-4, L261-L267, 2008.

Uyeda, S., Nagao, T., Orihara, Y., Yamaguchi, T., and Takahashi, I.: Geoelectric potential changes: Possible precursors to earthquakes in Japan, Proc. Nat. Acad. Sci. USA, 97, 9, 4561-4566, 2000.

Uyeda, S., Nagao, T., and Kamogawa, M.: Short-term earthquake prediction: Current status of seismo-electromagnetics, Tectonophysics, 470, 3-4, 205-213, 29 May 2009.

Vallianatos, F. and Triantis, D.: Scaling in Pressure Stimulated Currents related with rock fracture, Physica A, 387, 4940-4946, 2008.

Varotsos, P. and Alexopoulos, K.: Physical properties of the variations of the electric field of the earth preceding earthquakes, I, Tectonophysics 110, 73, 1984a.

Varotsos, P. and Alexopoulos, K.: Physical properties of the variations of the electric field of the earth preceding earthquakes. II. determination of epicenter and magnitude, Tectonophysics, 110, 99, 1984b.

Varotsos, P. and Alexopoulos, K.: Thermodynamics of Point Defects and their Relation with Bulk Properties, Noth-Holand, Amsterdam, 23-80, 1986.

Varotsos, P. A., Sarlis, N. V., and Skordas, E. S.: Practica of Athens 
Academy, 76, 294, 2001.

Varotsos, P. A., Sarlis, N. V., and Skordas, E. S.: Long-range correlations in the electric signals that precede rupture, Phys. Rev. E, 66, 011902, doi:10.1103/PhysRevE.66.011902, 2002.

Varotsos, P. A., Sarlis, N. V., and Skordas, E. S.: Attempt to distinguish electric signals of a dichotomous nature, Phys. Rev. E, 68, 031106, doi:10.1103/PhysRevE.68.031106, 2003.

Varotsos, P. A., Sarlis, N. V., Skordas, E. S., and Lazaridou M. S.: Entropy in natural time, Phys. Rev E, 70, 011106, doi:10.1103/PhysRevE.70.011106, 2004.

Varotsos, P. A., Sarlis, N. V., Skordas, E. S., and Lazaridou, M. S.: Natural entropy fluctuations discriminate similar looking electric signals emitted from systems of different dynamics, Phys. Rev. E, 71, 011110, doi:10.1103/PhysRevE.71.011110, 2005a.
Varotsos, P. A., Sarlis, N. V., Tanaka, H. K., and Skordas, E. S.: Some properties of the entropy in the natural time, Phys. Rev. E, 71, 032102, doi:10.1103/PhysRevE.71.032102, 2005b.

Varotsos, P. A., Sarlis, N. V., Skordas, E. S., Tanaka, H. K., and Lazaridou, M. S.: Entropy of seismic electric signals: Analysis in natural time under time reversal, Phys. Rev. E, 73, 031114, doi:10.1103/PhysRevE.73.031114, 2006.

Yépez, E., Angulo-Brown, F., Peralta, J. A., Pavia, C. G., and González-Santos, G.: Electric field patterns as seismic precursors, Geophys. Res. Lett., 22, 3087-3090, 1995. 